\title{
Prevalence of Depression and Anxiety Among Medical Students and House Staff During the COVID-19 Health-Care Crisis
}

\author{
Pratishtha Gupta ${ }^{1}$ Anupama B K ${ }^{1} \cdot$ Kartik Ramakrishna ${ }^{1}$ (D) \\ Received: 2 August 2020 / Accepted: 7 April 2021 / Published online: 20 May 2021 \\ (C) Academic Psychiatry 2021
}

\begin{abstract}
Objective The objective of the study was to evaluate the prevalence of anxiety and depression among medical trainees during the initial wave of the COVID-19 pandemic.

Methods This was a cross-sectional study of the prevalence of depression and anxiety among medical students, residents, and fellows at a medical university hospital in New York using self-reported PHQ-9 and GAD-7 screening tools administered via an anonymized survey. The study was conducted in April 2020.

Results The authors received 438 responses (33.4\% response rate). Nearly half (44.5\%) were medical students and female (56.6\%). The prevalence of positive screen for depression (45.3\%) and anxiety (48.1\%) was high. Many reported moderate to severe depression $(17.2 \%)$ and anxiety $(20.3 \%)$. Over half $(57.3 \%)$ experienced significant mood changes and inability to concentrate, and $14.6 \%$ had reconsidered their choice of profession since the start of the pandemic. Those who had reconsidered their profession had higher PHQ-9 [8.1 (6.4) vs 4.4 (4.3), $p<0.0001$ ] and GAD-7 scores [8.3 (6.1) vs 4.7 (4.6), $p<0.0001]$, indicating adverse mental health partly contributed to their reconsideration of choice of profession. Women were more likely to screen positive for anxiety (OR: 1.68) and medical students more likely to screen positive for anxiety (OR: 2.55$)$ and depression (OR: 2.74).

Conclusion The COVID-19 pandemic has placed great strain on health-care resources, including the mental health of medical trainees.
\end{abstract}

Keywords COVID-19 $\cdot$ Medical student $\cdot$ Resident $\cdot$ Anxiety $\cdot$ Depression

The COVID-19 pandemic has emerged as a health-care crisis of previously unimaginable magnitude. It was declared a public health emergency of international concern by the World Health Organization (WHO) on January 30,2020, and then a pandemic on March 11, 2020. The death toll of this pandemic has been high, more than 15,000 by early April 2020. There have been numerous reports of COVID infection among health-care workers in the media, with one report of almost 10,000 health-care workers having the infection by this time. During the early months of the pandemic, there was uncertainty about the rate and modes of transmission, with varying recommendations on appropriate protective measures. This was compounded by an unprecedented shortage of hospital

Kartik Ramakrishna

kartik_ramakrishna@hotmail.com

1 State University of New York Upstate Medical University, Syracuse, NY, USA beds, ventilators, health-care providers, and personal protective equipment for health-care providers. In New York State, the Governor even suggested lifting the 80 -h weekly work limit for residents.

It is well known that the prevalence of depression among medical students and resident physicians is well above that of the general population $[1,2]$. In large meta-analyses done prior to the COVID-19 pandemic, the pooled prevalence of depression was $27.2 \%$ among medical students and $28.8 \%$ among residents. The COVID-19 pandemic has taken a toll on the mental health of health-care providers. A recent study from Wuhan, China, reported a high prevalence of depression $(50.4 \%)$, anxiety $(44.6 \%)$, and insomnia $(34 \%)$ in healthcare professionals [3], with more severe depression in nurses, women, and frontline health-care workers. There are however no studies to date evaluating the effect of this health-care crisis on medical trainees, who are arguably the most vulnerable section of the health-care profession. 
We conducted this study to evaluate the prevalence of depression and anxiety on medical students and trainee physicians (resident physicians and subspecialty fellows) in light of the stresses induced by the COVID-19 pandemic.

\section{Methods}

The study is a cross-sectional survey of medical students, resident physicians, and subspecialty fellows at a university hospital in New York. The study was approved by our institutional review board and ethics committee. An email with details of the study along with a link to an anonymized survey was sent to the 662 medical students, 553 resident physicians, and 97 subspecialty fellows. The study period was from April 8 to April 25, 2020, and no responses after this period were included.

We designed an anonymous survey to obtain baseline demographic information and screen for depression and anxiety. The baseline demographic information obtained was role (medical student, resident, or fellow), gender, and whether they were directly involved in the care of patients with COVID-19 infection. We included choices for age group rather than requesting actual age in order to help maintain confidence in the anonymity of the survey. We screened for depression with the self-administered Patient Health Questionnaire-9 (PHQ-9) [4]. As outlined by Kroenke et al. [4], a score of 5 to 9 was considered positive for mild depression, 10 to 14 was considered consistent with moderate severity of depression, and 15 and above consistent with severe depression. We used the self-administered General Anxiety Disorder-7 (GAD-7) questionnaire to screen for and stratify severity of anxiety [5]. A score of 5 to 9 was considered positive for mild anxiety, 10 to 14 consistent with moderate severity, and 15 or more consistent with severe degree of anxiety. Other outcomes assessed included whether the trainee felt adequately supported through the COVID crisis, whether they had noticed recent significant mood changes or inability to concentrate, and whether the COVID-19 crisis had made them reconsider their choice of profession.

Statistical analysis was done using SPSS Version 16. Descriptive statistics were obtained for all study variables. All categorical variables were compared using Fisher's exact test and continuous variables using Student's $t$ test. This data was expressed as mean $( \pm \mathrm{SD})$, unless otherwise specified. Multinomial logistic regression was performed using baseline demographic data expressed as categorical variables, to look for independent associations with the presence of positive screen for depression and anxiety. Outcomes of multivariate analysis were expressed as odds ratio with $95 \%$ confidence interval. For all the statistical analysis, $P<0.05$ on two tailed analysis was considered statistically significant.

\section{Results}

By the time of closing this survey, there were over 350,000 confirmed cases of infection with SARS-CoV-2 in New York with over 28,000 deaths attributed to this infection. We received 438 responses (33.4\% response rate), 10 of which had completed the demographics section and PHQ-9 questions but had not completed the GAD-7 questionnaire. Half the responses $(49.5 \%)$ were from resident physicians, followed by medical students $(44.5 \%)$ and subspecialty fellows $(5.9 \%)$. The majority of respondents was females $(56.6 \%)$ and between the ages of 25 to $34(74.4 \%)$. The mean (SD) PHQ-9 and GAD-7 scores of each of these groups are detailed in Table 1. The difference in mean (SD) GAD-7 score between males and females was significant, 5.0 (5.4) vs 6.0 (5.0), $p=0.05$.

Almost half of the respondents screened positive for anxiety $(n=206,48.1 \%)$, with about a fifth screening positive for moderate to severe anxiety $(n=87,20.3 \%)$. The prevalence of positive screen for depression is similar $(n=198,45.3 \%)$, with over a sixth $(n=75,17.2 \%)$ screening positive for moderate to severe depression (Table 2). To the question "Have you noticed recent significant mood changes or inability to concentrate?", over half the trainees $(n=251,57.3 \%)$ reported having experienced these symptoms. When asked "Do you feel adequately supported through COVID-19 health crisis?", only 3 out of $4(n=325,74.2 \%)$ responded that the felt adequately supported. When asked "Has the COVID-19 crisis made you

Table 1 Baseline demographic data and associated PHQ-9 and GAD-7 score

\begin{tabular}{lll}
\hline $\begin{array}{l}\text { Characteristic } \\
N=\text { for PHQ-9, for GAD-7 }\end{array}$ & $\begin{array}{l}\text { PHQ-9 } \\
\text { Mean (SD) }\end{array}$ & $\begin{array}{l}\text { GAD-7 } \\
\text { Mean (SD) }\end{array}$ \\
\hline All respondents $(n=437,428)$ & $5.3(5.2)$ & $5.6(5.2)$ \\
Age groups & & \\
$18-24(n=74,74)$ & $5.9(4.8)$ & $6.4(5.4)$ \\
25-34 $(n=326,316)$ & $5.2(5.1)$ & $5.4(5.2)$ \\
$35-44(n=36,37)$ & $5.9(6.9)$ & $6.0(5.5)$ \\
$45+(n=1,1)$ & 0 & 0 \\
Gender & & \\
Female $(n=248,244)$ & $5.7(4.9)$ & $6.0(5.0)^{*}$ \\
Male $(n=185,181)$ & $5.0(5.5)$ & $5.0(5.4)^{*}$ \\
Role & & \\
Medical student $(n=195,189)$ & $5.8(4.4)$ & $6.1(4.9)$ \\
Resident $(n=216,214)$ & $5.1(5.8)$ & $5.2(5.5)$ \\
Fellow $(n=26,25)$ & $4.4(5.1)$ & $5.3(5.7)$ \\
Direct care of patients with COVID-19 & & \\
Yes $(n=168,165)$ & $5.5(5.8)$ & $5.6(5.7)$ \\
No $(n=269,263)$ & $5.3(4.8)$ & $5.5(5.0)$ \\
\hline
\end{tabular}

PHQ-9 Patient Health Questionnaire-9

$G A D-7$ General Anxiety Disorder-7

*GAD-7 female vs male $p=0.05$ 
Table 2 Prevalence of positive screen for depression, anxiety and secondary outcomes

\begin{tabular}{ll}
\hline Outcome & Prevalence $(\%)$ \\
\hline Depression & \\
None (PHQ-9 $=0-4)$ & $239(54.7 \%)$ \\
Mild (PHQ-9 $=5-9)$ & $123(28.1 \%)$ \\
Moderate (PHQ-9 $=10-14)$ & $20(4.6 \%)$ \\
Severe (PHQ-9 $\geq 15)$ & $55(12.6 \%)$ \\
Anxiety & \\
None (GAD-7 $=0-4)$ & $222(51.9 \%)$ \\
Mild (GAD-7 $=5-9)$ & $119(27.8 \%)$ \\
Moderate (GAD-7 $=10-14)$ & $51(11.9 \%)$ \\
Severe (GAD-7 $\geq 15)$ & $36(8.4 \%)$ \\
Mood changes since COVID outbreak & \\
Yes & $251(57.3 \%)$ \\
No & $187(42.7 \%)$ \\
Felt adequately supported through the crisis & \\
Yes & $325(74.2 \%)$ \\
No & $113(25.8 \%)$ \\
Reconsidered profession due to COVID outbreak & \\
Yes & $64(14.6 \%)$ \\
No & $374(85.4 \%)$ \\
\hline
\end{tabular}

PHQ-9 Patient Health Questionnaire-9

$G A D-7$ General Anxiety Disorder-7

re-consider your choice of profession?", many ( $n=64$, $14.6 \%$ ) admitted that the COVID-19 crisis had made them reconsider their choice of profession.

Interestingly, those who had reconsidered their choice of profession had higher PHQ-9 and GAD-7 scores that those who had not. The mean (SD) PHQ-9 scores were 9.0 (6.9) vs 4.7 (4.6), $p<0.0001$, and the mean (SD) GAD-7 scores were 10.4 (6.3) vs 4.8 (4.6), $p<0.0001$, respectively. Similarly, both the mean (SD) PHQ-9 and GAD-7 scores were higher among those who did not feel adequately supported. The mean (SD) PHQ-9 scores were 8.1 (6.4) vs 4.4 (4.3), $p<$ 0.0001 , and the mean (SD) GAD-7 scores were 8.3 (6.1) vs 4.7 (4.6), $p<0.0001$, respectively.

Table 3 compares and outlines the differences noted between medical students and house staff (resident physicians and subspecialty fellows). Medical students were noted to have a higher prevalence of depression $(n=109,55.9 \%$ vs $n$ $=91,37.4 \%, p=0.0002)$ and anxiety $(n=106,54.4 \%$ vs $n=$ $103,42.4 \%, p=0.0086)$ than house staff. They also reported significantly higher prevalence of mood change and inability to concentrate $(=138,70.8 \%$ vs $n=113,46.5 \%, p<0.0001)$ than house staff.

A number of differences were noted between males and females. The prevalence of positive screen for anxiety was higher in women $(n=133,54.5 \%)$ than men $(n=74$, $40.8 \%), p=0.006$. The difference in the prevalence of positive screen for depression between women and men showed a trend towards significance $(n=124,50 \%$ vs $n=76,41 \%, p$ $=0.08$ ). Women were more likely to report feeling inadequately supported ( $n=73,29.4 \%$ vs $n=38,20.4 \%, p=$ 0.035 ), and were more likely to report significant mood changes or inability to concentrate $(n=163,65.7 \%$ vs $n=$ $87,46.7 \%, p<0.0001)$. There was no difference in the number who had reconsidered their choice of profession between females and males $(n=37,14.9 \%$ vs $n=26,13.9 \%, p=0.89)$. There was no significant difference in the prevalence of depression and anxiety by age groups or by direct involvement in the care of patients with this infection.

The association between female gender and positive screen for anxiety remained on multivariate analysis (OR: 1.67, 95\% CI: 1.14-2.46). Multivariate analysis (Tables 4 and 5) also shows an independent association between current role and positive screen for anxiety and depression. Higher odds of positive screen were noted in medical students compared to residents for anxiety (OR: 2.55, 95\% CI: $1.42-4.58$ ) and depression (OR: 2.74, 95\% CI: 1.52-4.93).

\section{Discussion}

This was a cross-sectional study of the medical students and house staff of a medical university in New York with 438 respondents, performed during the COVID-19 pandemic. Depression and anxiety were diagnosed with a selfadministered PHQ-9 and GAD-7 anonymized questionnaire. The gold standard for diagnosing major depressive disorder and generalized anxiety disorder is through direct clinical interview and assessment. However, both the PHQ-9 and the GAD-7 have been shown to be reliable and valid tools to diagnose depressive disorders and generalized anxiety disorder as well as measure their severity $[4,5]$. Furthermore, concerns about inaccurate and dishonest responses in direct assessments of sensitive psychological states have been reported in medical trainees [6,7], perhaps making an anonymous survey more reliable. It must however also be noted that these tools were not validated for use in a pandemic. Some of the symptoms reported may be due to acute stress or grief from the pandemic that resolve with time, and these tools do not differentiate between acute and chronic symptoms.

It is known that the prevalence of depression and anxiety is higher among medical trainees than the general population under normal circumstances $[1,2]$. The effects of a healthcare crisis such as the COVID-19 crisis on this vulnerable population have not been reported on thus far. Lai et al. reported on the mental health outcomes of health-care workers exposed to the COVID-19 crisis in the Wuhan area of China [3]. They noted a high prevalence of symptoms of depression $(50.4 \%)$ and anxiety $(44.6 \%)$. They also noted more severe degrees of all measures of mental health outcomes in nurses, 
Table 3 Differences in prevalence of positive screen for depression and anxiety and other measures between medical students and house staff (residents and fellows)

\begin{tabular}{llll}
\hline Outcome & $\begin{array}{l}\text { Medical students } \\
N(\%)\end{array}$ & $\begin{array}{l}\text { House staff } \\
N(\%)\end{array}$ & $P$ value \\
\hline PHQ-9 positive for depression & $109(55.9 \%)$ & $91(37.4 \%)$ & 0.0002 \\
GAD-7 positive for anxiety & $106(54.4 \%)$ & $103(42.4 \%)$ & 0.0086 \\
Felt adequately supported & $152(77.9 \%)$ & $173(71.2 \%)$ & 0.124 \\
Experienced mood change & $138(70.8 \%)$ & $113(46.5 \%)$ & $<0.0001$ \\
Reconsidered their choice of profession & $24(12.3 \%)$ & $40(16.5 \%)$ & 0.276 \\
\hline
\end{tabular}

PHQ-9 Patient Health Questionnaire-9

GAD-7 General Anxiety Disorder-7

The questions asked were:

Do you feel adequately supported through COVID-19 health crisis?

Have you noticed recent significant mood changes or inability to concentrate?

Has the COVID-19 crisis made you re-consider your choice of profession? women, frontline workers, and those working in Wuhan, China.

Medical trainees in our cohort had a similarly high prevalence of positive screen for depression (45.3\%) and anxiety (48.1\%), with $17.2 \%$ and $20.3 \%$ screening positive for moderate to severe depression and anxiety, respectively. The baseline prevalence of depression and anxiety in this specific cohort prior to the COVID-19 pandemic is not known. However, previous meta-analysis of 54 studies involving 17,560 individuals, by Mata D et al. [2], showed the estimated pooled prevalence of depression among residents to be $28.8 \%$, with a $20.9 \%$ prevalence of moderate to severe depression by PHQ-
9. Similarly, a meta-analysis of 195 studies involving 129,123 individuals, by Rotenstein et al. [1], showed a pooled global prevalence of $27.2 \%$, with a prevalence of $26.7 \%$ in studies from North America. They reported an $18.3 \%$ prevalence of moderate to severe depression by PHQ-9.

Furthermore, more than half the trainees $(57.3 \%)$ reported having had recent significant mood changes or inability to concentrate since the start of the COVID-19 crisis. One in four trainees felt inadequately supported, and about a sixth admitted to having reconsidered their choice of profession since the start of the pandemic in America. All of these suggest that this pandemic was contributing significantly and adversely to their
Table 4 Multivariate analysis of association between positive screen for depression and gender, responsibility for direct care of COVID patients, age group, and current role of the respondent

\begin{tabular}{|c|c|c|c|c|c|c|c|}
\hline \multirow[t]{3}{*}{ Variable } & \multicolumn{2}{|c|}{ Depression } & \multirow[t]{3}{*}{$P$ (univariate) } & \multicolumn{4}{|c|}{ Multivariate analysis } \\
\hline & \multirow[t]{2}{*}{ Absent } & \multirow[t]{2}{*}{ Present } & & \multirow[t]{2}{*}{ Odds ratio } & \multicolumn{2}{|c|}{$95 \% \mathrm{CI}$} & \multirow[t]{2}{*}{$P$ (multivariate) } \\
\hline & & & & & Lower & Upper & \\
\hline \multicolumn{8}{|l|}{ Gender } \\
\hline Female & 125 & 133 & 0.053 & 1.287 & 0.879 & 1.884 & 0.195 \\
\hline Male & 113 & 87 & & & & & \\
\hline \multicolumn{8}{|l|}{ Direct care } \\
\hline No & 140 & 141 & 0.144 & 0.644 & 0.376 & 1.104 & 0.110 \\
\hline Yes & 98 & 79 & & & & & \\
\hline \multicolumn{8}{|l|}{ Age interval } \\
\hline $18-24$ & 35 & 40 & 0.587 & 0.585 & 0.245 & 1.398 & 0.228 \\
\hline $25-34$ & 182 & 160 & & 0.711 & 0.359 & 1.406 & 0.327 \\
\hline$\geq 35$ & 21 & 20 & & & & & \\
\hline \multicolumn{8}{|l|}{ Current role } \\
\hline Fellow & 15 & 12 & 0.004 & 1.162 & 0.511 & 2.644 & 0.720 \\
\hline Med student & 87 & 114 & & 2.745 & 1.528 & 4.932 & 0.001 \\
\hline Resident & 136 & 94 & & & & & \\
\hline
\end{tabular}

All variables were categorical. Odds ratios and $95 \%$ confidence intervals are presented for association of positive screen for depression, against negative screen for depression as the reference category 
Table 5 Multivariate analysis of association between positive screen for anxiety and gender, responsibility for direct care of COVID patients, age band, and current role of the respondent

\begin{tabular}{|c|c|c|c|c|c|c|c|}
\hline \multirow[t]{3}{*}{ Variable } & \multicolumn{2}{|l|}{ Anxiety } & \multirow[t]{3}{*}{$P$ (univariate) } & \multicolumn{4}{|c|}{ Multivariate analysis } \\
\hline & \multirow[t]{2}{*}{ Absent } & \multirow[t]{2}{*}{ Present } & & \multirow[t]{2}{*}{ Odds ratio } & \multicolumn{2}{|c|}{$95 \% \mathrm{CI}$} & \multirow[t]{2}{*}{$\mathrm{P}$ (multivariate) } \\
\hline & & & & & Lower & Upper & \\
\hline \multicolumn{8}{|l|}{ Gender } \\
\hline Female & 102 & 156 & 0.002 & 1.677 & 1.143 & 2.460 & 0.008 \\
\hline Male & 107 & 93 & & & & & \\
\hline \multicolumn{8}{|l|}{ Direct care } \\
\hline No & 122 & 159 & 0.248 & 0.664 & 0.390 & 1.131 & 0.132 \\
\hline Yes & 87 & 90 & & & & & \\
\hline \multicolumn{8}{|l|}{ Age interval } \\
\hline $18-24$ & 30 & 45 & 0.325 & 0.439 & 0.179 & 1.076 & 0.072 \\
\hline $25-34$ & 163 & 179 & & 0.516 & 0.256 & 1.039 & 0.064 \\
\hline$\geq 35$ & 16 & 25 & & & & & \\
\hline \multicolumn{8}{|l|}{ Current role } \\
\hline Fellow & 14 & 13 & 0.007 & 0.961 & 0.421 & 2.195 & 0.961 \\
\hline Med student & 75 & 126 & & 2.554 & 1.422 & 4.589 & 0.002 \\
\hline Resident & 120 & 110 & & & & & \\
\hline
\end{tabular}

All variables were categorical. Odds ratios and $95 \%$ confidence intervals are presented for association of positive screen for anxiety, against negative screen for anxiety as the reference category mental health. Interestingly, those who had reconsidered their choice of profession had markedly higher PHQ-9 and GAD-7 scores than the other survey participants, suggesting that the adverse mental health effects of this crisis were playing a role in reconsideration of their choice of profession.

Multiple studies have shown a higher prevalence of self-reported depressive symptoms in female than male medical students and residents [8-10]. In our cohort as well, more women reported symptoms of anxiety $(54.5 \%$ vs $40.8 \%, p=0.006)$, depression ( $50 \%$ vs $41 \%, p=0.08$ ), not feeling adequately supported and having mood changes or inability to concentrate $(65.7 \%$ vs $46.7 \%, p<$ $0.0001)$. This may be because women are more aware of their mental health than men and hence more likely to report it [11]. That the number of women who had reconsidered their choice of profession was no higher than men would support this premise.

We anticipated the prevalence of depression and anxiety to be higher among house staff than the medical students, as they were more likely to be directly involved in patient care and more likely to be exposed to the infection and increased workload. However, we found a significantly higher prevalence of depression, anxiety, and mood change among medical students. The reasons behind this are unclear. Anxiety about the temporary closure of the medical school, and suspension of on-site classes and education, hampering the quality of their education, as well as concerns about effects on matching to a residency program may have contributed to this.
As discussed earlier, there are several limitations to this study. The first being that baseline data on the prevalence of symptoms of depression and anxiety in our cohort prior to the COVID-19 pandemic are not available. While the effects on mental health were determined by a self-reported survey, as discussed earlier, this may be more optimal than direct interview in this cohort. Lastly, this is a single-center experience, and the effects of this pandemic may vary at medical centers in other parts of the country.

The COVID-19 pandemic has placed a great strain on society at large and on health-care systems and providers in particular. There is an increased demand on health-care resources and increased need for health-care providers. In our study of medical trainees, we found a high prevalence of depression and anxiety, partly attributable to the ongoing pandemic, with many admitting to reconsidering their choice of profession. Medical trainees are very vulnerable to the adverse mental health effects of such a crisis. If we are to respond effectively to this and subsequent healthcare crises, we need to be mindful of their effects on trainees and adopt measures to mitigate these harmful effects on their mental health.

\section{Declarations}

Disclosures On behalf of all authors, the corresponding author states that there is no conflict of interest. 


\section{References}

1. Rotenstein LS, Ramos MA, Torre M, Segal JB, Peluso MJ, Guille $\mathrm{C}$, et al. Prevalence of depression, depressive symptoms, and suicidal ideation among medical students: a systematic review and meta-analysis. JAMA. 2016;316(21):2214-36.

2. Mata DA, Ramos MA, Bansal N, Khan R, Guille C, di Angelantonio E, et al. Prevalence of depression and depressive symptoms among resident physicians: a systematic review and meta-analysis. JAMA. 2015;314(22):2373-83.

3. Lai J, Ma S, Wang Y, Cai Z, Hu J, Wei N, et al. Factors associated with mental health outcomes among health care workers exposed to coronavirus disease 2019. JAMA Netw Open. 2020;3(3):e203976.

4. Kroenke K, Spitzer RL, Williams JB. The PHQ-9: validity of a brief depression severity measure. J Gen Intern Med. 2001;16(9):60613.

5. Spitzer RL, Kroenke K, Williams JB, et al. A brief measure for assessing generalized anxiety disorder: the GAD-7. Arch Intern Med. 2006;166(10):1092-7.

6. Levine RE, Breitkopf CR, Sierles FS, Camp G. Complications associated with surveying medical student depression: the importance of anonymity. Acad Psychiatry. 2003;27(1):12-8.
7. Myers M. On the importance of anonymity in surveying medical student depression. Acad Psychiatry. 2003;27(1):19-20.

8. Dahlin M, Joneborg N, Runeson B. Stress and depression among medical students: a cross-sectional study. Med Educ. 2005;39(6): 594-604.

9. Peterlini M, Tibério IF, Saadeh A, et al. Anxiety and depression in the first year of medical residency training. Med Educ. 2002;36(1): 66-72.

10. Tabalipa F, Souza M, Pfützenreuter G, et al. Prevalence of anxiety and depression among medical students. Rev Bras Educ Méd. 2015;39(3):388-94.

11. Christiansen DM. Examining sex and gender differences in anxiety disorders, a fresh look at anxiety disorders, Federico Durbano, IntechOpen. 2015. https://doi.org/10.5772/60662. Available from: https://www.intechopen.com/books/a-fresh-look-at-anxietydisorders/examining-sex-and-gender-differences-in-anxietydisorders. Accessed 20 May 2020.

Publisher's Note Springer Nature remains neutral with regard to jurisdictional claims in published maps and institutional affiliations. 